\title{
GlyT2/5HT2A Antagonist VVZ-149
}

National Cancer Institute

\section{Source}

National Cancer Institute. GlyT2/5HT2A Antagonist VVZ-149. NCI Thesaurus. Code C123910.

An antag onist of both glycine transporter type 2 (GlyT 2) and serotonin receptor $2 \mathrm{~A}$ (5HT2A), with potential anti-nociceptive activity. Upon administration, GlyT 2/5HT2A antagonist VVZ-149 binds to and blocks both GlyT2 and 5HT2A. Blockage of GlyT2 prevents the re-uptake of the inhibitory neurotransmitter glycine in the synaptic cleft, thereby potentiating glycine-mediated inhibitory signaling, and inhibiting the firing of neurons, which suppresses the transmission of pain signals to the brain and induces analgesia. Blockage of 5HT2A prevents both the binding of its ligand serotonin and 5HT/5HT2A-mediated signaling. This also suppresses pain signaling and induces analgesia. GlyT 2 and 5HT2A play key roles in the induction and transmission of pain signals. GlyT2, a glycine plasma membrane transporter, modulates glycine-mediated inhibition of synaptic transmission in the spinal cord and mediates pain signal transmission to the brain; inhibition of GlyT 2 potentiates glycinergic mediated pain signaling. Serotonin and its 5HT2A receptor are involved in both serotonin receptormediated signaling and the perception of pain. 